\title{
NOVEL MUTATIONS IN THE ANOCTAMIN 5 GENE (ANO5) ASSOCIATED WITH LIMB-GIRDLE MUSCULAR DYSTROPHY 2L
}

ANN A. LITTLE, MD, ${ }^{1}$ PAUL E. MCKEEVER, MD, PhD, ${ }^{2}$ and KIRSTEN L. GRUIS, MD, MS ${ }^{3}$

${ }^{1}$ Department of Neurology, University of Michigan Medical Center, 1500 E. Medical Center Drive, 1 C327 UH, EMG Lab, Ann Arbor, Michigan 48109, USA

${ }^{2}$ Department of Pathology, University of Michigan Medical Center, 1500 E. Medical Center Drive, Ann Arbor, Michigan 48109, USA

${ }^{3}$ Department of Neurology, SUNY Upstate Medical University, 750 E. Adams Street, Syracuse, New York 13210, USA

Accepted 28 July 2012

ABSTRACT: Introduction: We present a Jordanian man with the typical LGMD 2L phenotype of early, asymmetric quadriceps weakness and subsequent biceps brachii weakness. Methods: Case report. Results: Muscle biopsies document a progressive dystrophic pattern unrelated to known sarcolemmal defects associated with muscular dystrophy. Genetic testing revealed novel, heterozygote Anoctamin 5 gene mutations. Conclusions: This case report expands the known mutations resulting in LGMD $2 \mathrm{~L}$ and supports the assertion that Anoctamin 5 mutations are more prevalent than previously recognized. Muscle Nerve 47: 287-291, 2013

The original description of the Anoctamin 5 (ANO5) mutation associated with limb-girdle muscular dystrophy (LGMD) 2L was published in 2007. ${ }^{1}$ Jarry et al. described 14 patients from 8 families of French Canadian ancestry. ${ }^{1}$ Since then, ANO5 mutations associated with muscular dystrophy-both LGMD 2L and Miyoshi-like muscular dystrophy type 3 (MMD3) - have been described in more diverse European populations. ${ }^{2-4}$

The phenotype of LGMD 2L includes adult onset (ages, 20-50 years; mean, 30 years) with slow progression of asymmetric quadriceps and biceps brachii muscle atrophy and weakness. Quadriceps involvement precedes biceps brachii weakness, and creatine kinase $(\mathrm{CK})$ is $>10 \times$ the upper limit of normal (mean 4,500 IU/L). No significant cardiac or respiratory muscle involvement has been reported. ${ }^{1,3,5}$

A recent publication suggests a founder effect of the truncating mutation in the Northern European population. ${ }^{3}$ Furthermore, Hicks et al. report LGMD 2L may be twice as prevalent as dysferlinopathy (LGMD 2B), and, therefore, it is a common type of adult-onset muscular dystrophy in those of European ancestry. ${ }^{3}$

We report a novel mutation in ANO5 associated with an LGMD 2L phenotype in a man of Jordanian ancestry. This case illustrates a new mutation in ANO5 associated with the LGMD 2L phenotype.

Abbreviations: ANO, anoctamin; CK, creatine kinase; EMG, electromyography; IU/L, International units/liter; LGMD, Limb Girdle Muscular Dystrophy; MMD3, Miyoshi muscular dystrophy type 3

Key words: Anoctamin 5; ANO5; creatine kinase; limb-girdle muscular dystrophy; mutation; asymmetric weakness

Correspondence to: A. A. Little; e-mail: alitt@med.umich.edu

(C) 2012 Wiley Periodicals, Inc.

Published online 1 August 2012 in Wiley Online Library (wileyonlinelibrary. com). DOI 10.1002/mus.23542
Additionally, this case demonstrates LGMD 2L in a man of non-European ancestry.

\section{CASE REPORT}

A 37-year-old man presented in December 2002 with a chief complaint of right leg weakness for the past year. He reported that his right knee would give out, leading to unexpected falls and difficulty climbing stairs. He had no sensory symptoms, fasciculations, or pain. He had known left foot drop reported to be secondary to a lumbar radiculopathy in 1991, which was treated with lumbar discectomy and fusion at that time.

Past medical history included Graves disease in 2000, which was treated successfully with radioablation of the thyroid.

Neither of his deceased parents had a history of muscle weakness. He currently has 10 siblings and 45 nieces and nephews without similar weakness. He is originally from Jordan. He moved to the United States in 1998. He can trace his ancestry back to the early 1800s and knows of no European ancestors and no consanguinity.

On physical examination in December 2002, he had normal muscle bulk, tone, and strength in all muscle groups of the upper extremities. In the lower extremities, he had bilateral, distal posterior leg and right quadriceps muscle atrophy. Muscle tone was normal. His strength-right over left as graded by medical research council (MRC) scalewas: hip flexors $4 / 4+$, knee extensors $4 / 5$, knee flexors 5/5, ankle dorsiflexion 5/4 (weakness thought to be from 1991 radiculopathy), foot eversion $5 / 5$, foot inversion $5 / 5$, and plantar flexion $5-/ 5-$. There was no facial or neck flexion weakness. Tendon reflexes were $2+$ at bilateral biceps brachialis and patellar tendons, but they were absent at the Achilles tendons. Sensation was preserved to light touch, pinprick, vibration, and proprioception bilaterally. Electrodiagnostic testing demonstrated normal nerve conduction studies of bilateral sural and left ulnar sensory and bilateral fibular and tibial and left ulnar motor nerves. Concentric needle electromyography (EMG) demonstrated abnormal spontaneous activity in the form of positive sharp waves and fibrillation 


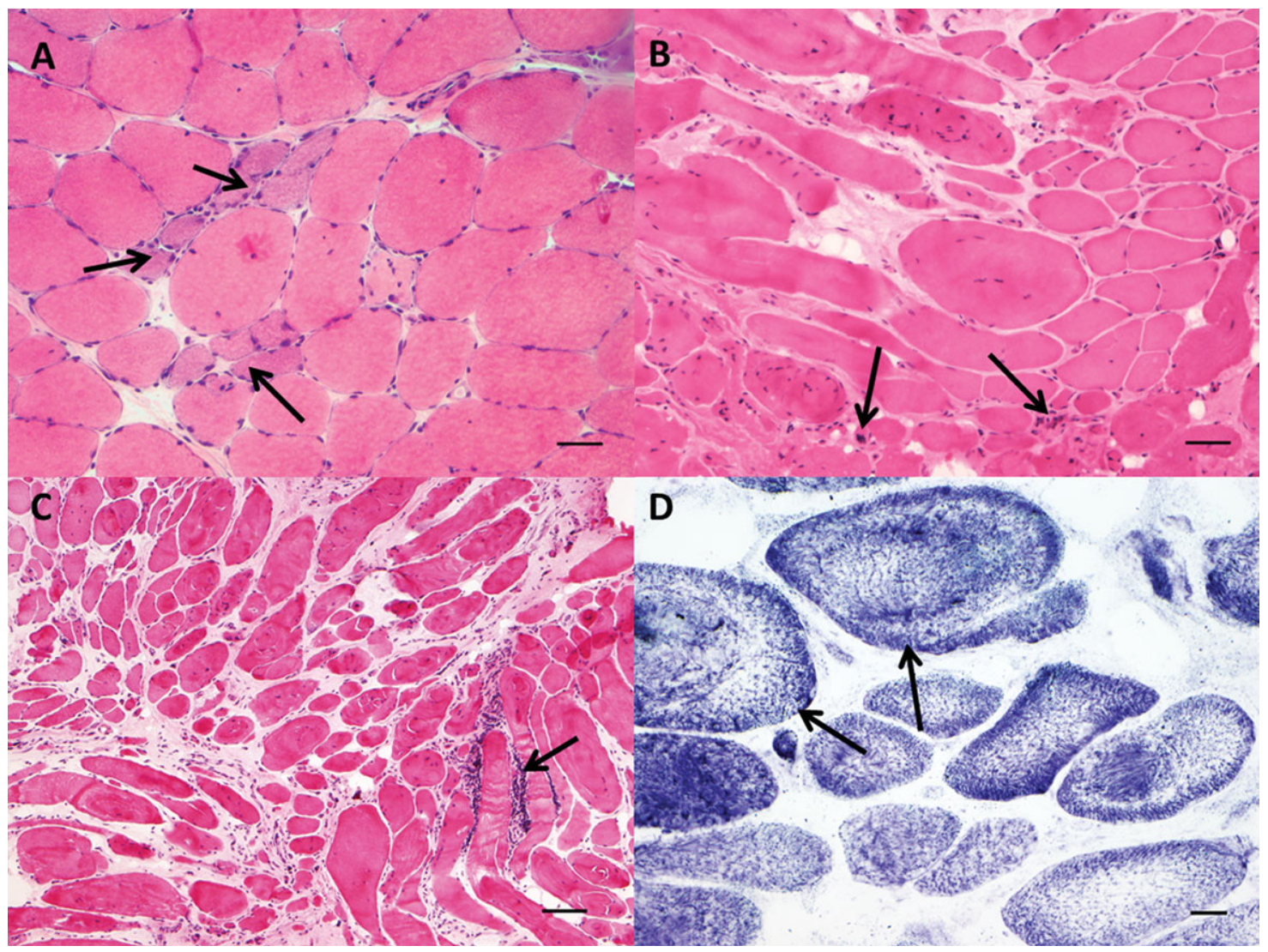

FIGURE 1. Light microscopy of left vastus lateralis muscle biopsies. (A) Biopsy of left vastus lateralis in 2003 shows myopathic features including muscle fiber degeneration and regeneration (arrows). An increase in internalized nuclei, slight fiber hypertrophy and fiber splitting are present. Fiber sizes vary from less than 30 to 100 microns. Scale bar $=50$ microns. (B) Biopsy of left vastus lateralis in 2006 shows marked increase in endomysial collagen and fiber size variation (less than 10 to 115 microns). Some of the largest fibers are whorled fibers. Internalized nuclei are more frequent, and many individual fibers have greater than 2 Internalized nuclei. Nuclear myobags (arrows) are present. Scale bar $=50$ microns. (C) Endomysial inflammation (arrow) is prominent, 2006. Scale bar $=$ 100 microns. (D) Succinic dehydrogenase stain highlights large whorled fibers (arrows), 2006. Scale bar $=20$ microns.

potentials in bilateral vastus medialis, right vastus lateralis, left anterior tibialis, left first dorsal interosseus of the hand, and thoracic paraspinal muscles. Voluntary motor unit potentials in limb muscles were all of decreased amplitude and increased recruitment, associated with polyphasia. An MRI of the lumbosacral spine revealed no evidence of lumbar root impingement or central canal stenosis. Mild bilateral foraminal canal stenosis was seen at the L5-S1 level. The CK was found to be elevated at $5303 \mathrm{IU} / \mathrm{L}$. An evaluation of inflammatory and autoimmune markers was negative and included anti-nuclear antibody, anti-Ro antibody, anti-La antibody, anti-Smith antibody, anti-ribonucleoprotein antibody, perinuclear anti-neutrophil cytoplasmic antibody, cytoplasmic anti-neutrophil cytoplasmic antibody, Westergren sedimentation rate, liver smooth muscle antibody, and liver mitochondrial antibody.

Muscle biopsy of the left vastus lateralis was completed in February 2003. The left vastus lateralis was chosen, because the right vastus lateralis had recently undergone EMG study and because electrodiagnostic involvement of the left vastus medialis suggested diffuse quadriceps involvement. Light microscopy of muscle tissue revealed degenerating myofibers and clusters of regenerating myofibers. Other abnormalities included frequent split fibers, variable muscle fiber diameter, whorled fibers, and internalized nuclei (Fig. 1A). Some fibers were surrounded and invaded by inflammatory cells, and rare muscle fibers had small vacuoles (reported but not pictured here). Standard muscle stains of Gomori trichrome, Congo red, oil red $\mathrm{O}$, PAS with and without diastase revealed no further abnormalities. Histochemical reactions (ATPase at $\mathrm{pH} 9.4,4.6$, and 4.2; toluidine blue, myophosphorylase, myoadenylate deaminase, phosphofructokinase, NADH-TR, cytochrome C oxidase $[\mathrm{CCO}]$, succinic dehydrogenase $[\mathrm{SDH}]$, combined $\mathrm{CCO} / \mathrm{SDH}$, alkaline and acid phosphatases, nonspecific esterase) also revealed no further abnormalities than those noted above. Additional assessment for a muscular dystrophy through immunohistochemical staining was pursued and was negative; immunohistochemical stains included 


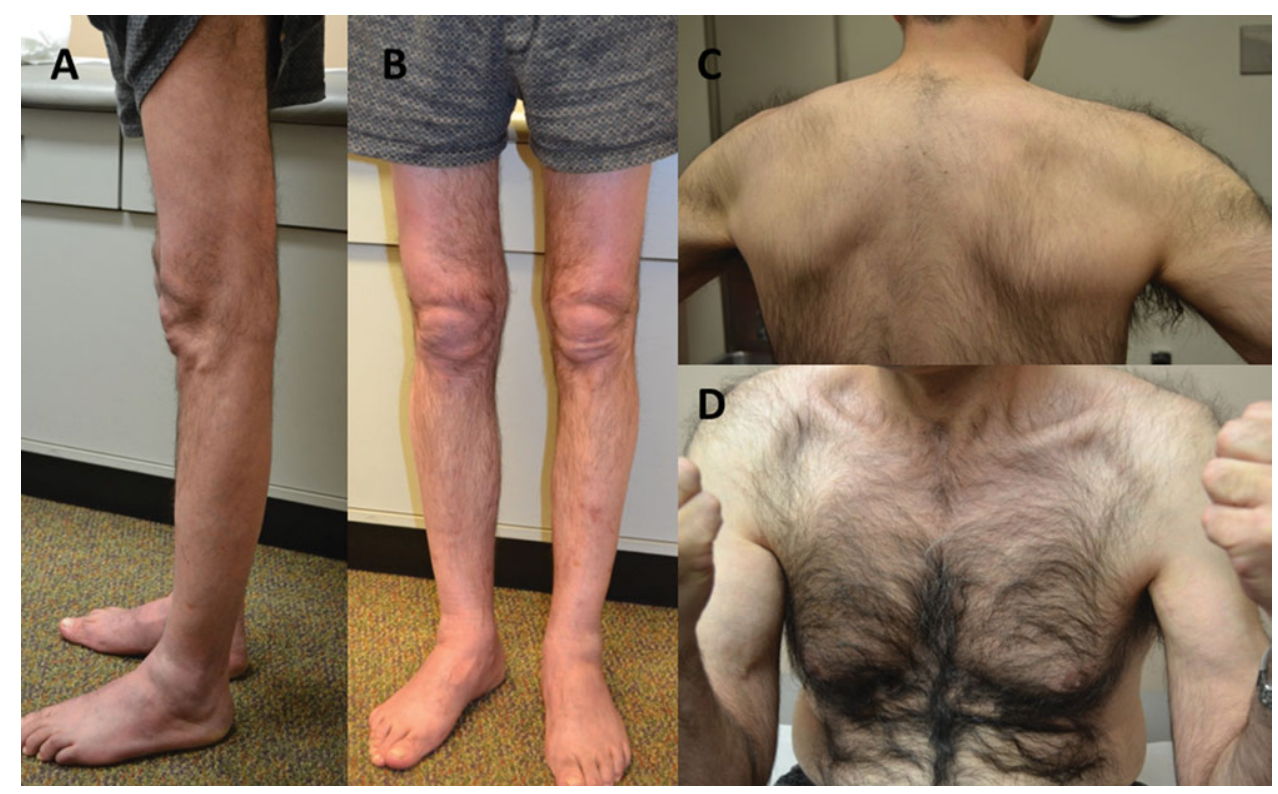

FIGURE 2. Muscular atrophy—patient photos. (A) Atrophy of anterior and posterior compartments of lower leg, quadriceps and hamstring muscles. (B) Quadriceps atrophy. (C) Atrophy of shoulder girdle muscles. (D) Atrophy of biceps and pectoral muscles: the patient's left upper limb is more affected than the right.

dysferlin, caveolin 3, sarcoglycans (alpha, beta, delta, gamma), dystrophin, emerin, merosin, laminin, lamin A/C, myotilin, and beta-dystroglycan, and all were equivalent to that of control muscle.

No further testing for muscular dystrophy was done, and the patient began treatment for a possible idiopathic inflammatory myopathy in April 2003. He was treated initially with oral prednisone $60 \mathrm{mg}$ daily, and it was later combined with oral methotrexate up to $25 \mathrm{mg}$ weekly. He continued to progress slowly with increasing weakness of his right quadriceps. His CK remained elevated, typically between 3,000 and $6,000 \mathrm{IU} / \mathrm{L}$ with an outlier of 9,000 IU/L after exercise.

We initially met and evaluated the patient in 2006. His examination at that time continued to show full strength in facial muscles, neck flexors, and upper extremity muscles. Bilateral quadriceps atrophy was now present, and he had asymmetrical knee extension and flexion weakness, greater on the right. Bilateral dorsiflexion weakness was noted. Reflexes were $2+$ at biceps brachii, $1+$ at patellae, and absent at the Achilles tendon. Sensation remained intact to multiple modalities as recorded before. We questioned the utility of continuing immunosuppression given his progressive weakness and muscle atrophy with continued CK elevation. We performed a repeat biopsy of the left vastus lateralis muscle in August 2006. The findings were consistent with muscular dystrophy with marked myofiber replacement (up to $50 \%$ ) by connective tissue (Fig. 1B and C). Inflammatory infiltrates were still seen, as was a more progressive stage of myofiber size variability and splitting (Fig.
1C). Large whorled fibers were present. Several CCO-negative fibers were seen. The pathological progression of dystrophic changes and lack of response to immunosuppressive therapy led to further testing for muscular dystrophies. Sequencing of genes for fukutin-related protein, calpain-3, lamin A/C, caveolin-3, alpha-sarcoglycan and dystrophin were normal. Monocyte dysferlin levels were normal. Genetic testing for facioscapulohumeral muscular dystrophy (FSHD) was negative. He continued to be followed in our clinic for the diagnosis of unspecified muscular dystrophy and developed symptoms of prominent and progressive quadriceps weakness with bilateral foot drop and plantar flexion weakness. In 2009 he developed mild weakness of bilateral biceps brachii. Motor examination in November 2011 revealed muscle atrophy in the bilateral supraspinati, pectoral, and biceps brachii muscles in the arms and in bilateral quadriceps, hamstrings, and anterior and posterior muscles in the lower legs (Fig. 2). On strength testing, there was mild weakness of bilateral biceps brachii and supraspinati with preserved function in the deltoid, triceps, and distal upper extremity muscles. Lower extremity weakness was graded as follows $(\mathrm{R} / \mathrm{L})$ : hip flexion 4/4, knee extension 2/ 2 , knee flexion $4 / 3$, dorsiflexion $3 / 0$, and plantar flexion 4/4. The patient remained ambulatory without an assistive device, but had significant difficulty arising from a seated position, marked difficulty with steps, and significant hyperextension at both knees with casual gait. He had no symptoms of cardiac or respiratory dysfunction. CK levels remain elevated at approximately 3,000-4,000 IU/ 


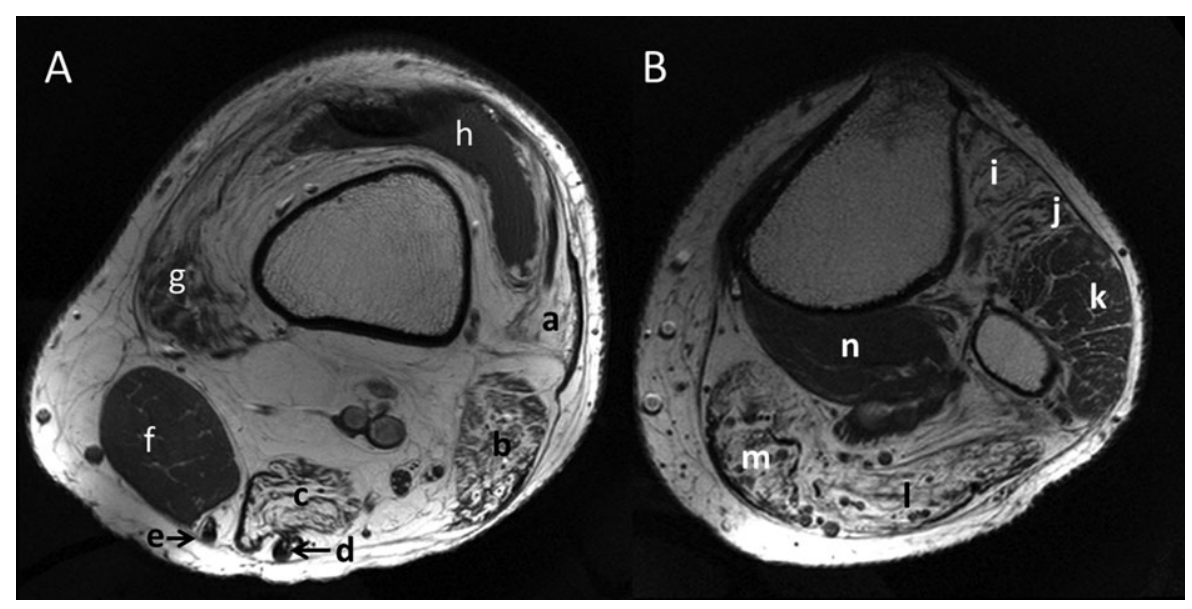

FIGURE 3. Muscle atrophy - MRI imaging. (A) MRI of left thigh in cross section shows severe muscle atrophy with relative sparing of the sartorius muscle (a, vastus lateralis; b, biceps femoris; c, semimembranosus; d, semitendinosus; e, gracilis; $f$, sartorius; $g$, vastus medialis; h, joint effusion). (B) MRI of left lower leg in cross section shows severe muscle atrophy (i, tibialis anterior; j, extensor digitorum longus; $k$, fibularis longus and brevis; I, lateral head of gastrocnemius; $m$, medial head of gastrocnemius; $n$, popliteus).

L. Of interest, he incidentally had an MRI of his left knee after a motor vehicle accident in 2008. Note was made of severe atrophy of quadriceps, hamstrings, gastrocnemius, tibialis anterior, and extensor digitorum longus muscles with relative sparing of the sartorius, popliteus, and fibularis longus and brevis muscles (Fig. 3).

Given his evolving phenotype of early asymmetric quadriceps weakness, later biceps brachii weakness, and high $\mathrm{CK}$, he underwent ANO5 gene sequencing by means of Prevention Genetics, Marshfield WI, USA in September 2011. Genomic DNA was amplified and sequenced of the full coding regions of the ANO5 exons and approximately 50 base pairs flanking the noncoding sequences. Our patient was found to have 2 heterozygous ANO5 mutations that were previously unreported. One mutation, c.352delG, is expected to result in a frameshift mutation with early stop codon (p.Glu118LysfsStop23) and subsequent truncation of the anoctamin protein. The other mutation is a base pair substitution, c. $1025 \mathrm{G}>\mathrm{A}$, on exon 11 of the ANO5 gene that results in an amino acid substitution.

\section{DISCUSSION}

ANO5 codes for a member of the anoctamin family of proteins, which comprise 10 members. The structure of anoctamin is that of a transmembrane protein with 8 hydrophobic transmembrane segments with 1 re-entry loop, cytosolic $\mathrm{N}$ and $\mathrm{C}$ terminals, and a relatively preserved domain of unknown function (DUF). Transmembrane segments contain the most-conserved sequences of amino acids. Ion channel function is postulated for the anoctamin family. ANO1 and ANO2 are known calcium-activated chloride channels; ANO6 and ANO7 produce chloride currents; $A N O 9$ and
ANO10 suppress chloride currents under certain conditions. ${ }^{6}$ The anoctamins have diverse roles in various tissues and are believed to play a role in gut motility, vascular smooth muscle function, nociception, sensory transduction, metastasis in several cancers, musculoskeletal disorders (muscular dystrophy and skeletal development), blood clotting and cerebellar ataxia. ${ }^{7}$

An ANO5 (GDD1) mutation underlies the autosomal dominant skeletal disorder of gnathodiaphyseal dysplasia, which is characterized by skeletal abnormalities, fibro-osseus lesions of jawbones, and predisposition to jaw infection and recurrent osteomyelitis. Investigation of this disease has led to interesting insight into ANO5 expression in muscle, noting myotomal expression of ANO5 (GDD1) during mouse development and upregulation of ANO5 (GDD1) protein levels in dystrophin-defi-

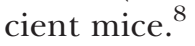

$A N O 5$ is predicted to have several alternatively spliced isoforms. ANO5 mutations are known to result in autosomal recessive muscular dystrophies of 2 phenotypes: LGMD 2L and MMD3. ${ }^{5}$ Some authors describe LGMD 2L and MMD3 as separate phenotypes, where MMD3 presents with calf atrophy and plantar flexion weakness starting before asymmetrical quadriceps weakness, while LGMD 2L presents with asymmetric quadriceps and biceps brachii atrophy and weakness with mild plantar flexion weakness. ${ }^{5}$ Other authors note that initial symptoms of predominant distal or proximal weakness may vary, but LGMD 2L and MMD3 phenotypes overlap substantially over time. ${ }^{3}$ Hicks et al. also report that $85 \%$ of LGMD 2L patients may have early difficulty walking on toes with calf atrophy. Because our patient had only mild plantar flexion weakness at the time of significant, asymmetrical quadriceps weakness, we believe he falls 
into the LGMD 2L phenotype. The LGMD 2L phenotype has some overlap with dysferlinopathy (LGMD 2B) with the combination of both distal and proximal lower limb weakness. Dysferlinopathy is believed to result from defective participation of dysferlin in the sarcolemmal repair process responsible for maintaining the sarcolemma in the face of injuries imposed by high mechanical stress. Electron microscopy in an LGMD 2L patient and a MMD3 patient have demonstrated multifocal disruption of the sarcolemmal membrane. Defective membrane repair was demonstrated in fibroblasts of a person with MDD3., ${ }^{2,5}$ Thus, defective sarcolemmal membrane repair has been suggested as a possible mechanism for ANO5 associated myopathies.

Muscle biopsies of patients with LGMD 2L and MMD3 have demonstrated dystrophic changes with increase in endomysial connective tissue, muscle fiber size variation, degeneration and regeneration of muscle fibers, internalized nuclei, and fiber splitting. Mild focal inflammation was noted in a case of a severely affected patient, although a larger study demonstrated inflammatory infiltrates in 6 of $19(31 \%)$ patients. ${ }^{1,3}$ Rimmed vacuoles have been noted in 2 patients, and congophilic deposits in intramuscular blood vessels and the interstitium were seen in another patient. ${ }^{3,9}$ Exclusions have been deficiencies in dystrophin, dysferlin, and sarcoglycans. Electron microscopy in 2 separate reports has demonstrated reduplicated basement membrane associated with multifocal loss of sarcolemmal membrane., ${ }^{1,2}$

The most commonly reported genetic mutation producing LGMD 2L is a truncating mutation, c.191dupA in exon 5; it has been seen in 20 of 64 patients from Germany and the United Kingdom, with homozygosity in 15 of the patients and compound heterozygosity in another 5. This mutation may result from a founder effect in the Northern European population. ${ }^{3}$ Several other mutations in ANO5 have also been reported, many in combination with the c.191dupA mutation, which is believed to produce disease when it is homozygous or compound heterozygous with other mutations. ${ }^{2,3,5,9}$ Like c.191dupA, our patient's heterozygous ANO5 mutation, c.352delG, also predicts a frame shift mutation and protein truncation. Therefore, given our patient's phenotype, the novel c.352delG heterozygote mutation on exon 6 of the ANO5 gene is likely associated with LGMD 2L.

Additionally, our patient also had a heterozygous base pair substitution, c.1025G $>$ A on exon 11 of the ANO5 gene. This mutation results in an amino acid substitution p.Cys342Tyr. The p.Cys342 residue is in a known, highly conserved region of the anoctamin- 5 protein across humans, primates, and nonprimate mammals, as well as the lizard, chicken, frog, and fish. Loss of a cysteine amino acid would alter disulfide bonds known to be crucial for proper protein folding. Further analysis of this amino acid substitution by Prevention Genetics, Marshfield, WI using the program PolyPhen-2 predicts the p.Cys342Tyr substitution to be "probably damaging" to the function of anoctamin- $5 .{ }^{10}$ Therefore, given our patient's phenotype, the novel c. $1025 \mathrm{G}>\mathrm{A}$, heterozygous ANO5 mutation is also likely to be associated with LGMD 2L. To our knowledge this is the first patient with LGMD 2L phenotype and compound heterozygous ANO5 mutations reported in an individual with non-European ancestry. Initially described in several FrenchCanadian families, LGMD 2L with underlying ANO5 mutations is now recognized in Northern European populations, and its prevalence may be relatively high among the more common LGMD syndromes. Our case underscores that LGMD 2L may be present in an even broader population than is currently recognized, and further diseasecausing ANO5 mutations may come to light. Screening for ANO5 is recommended in cases with the appropriate phenotype, given the potential prevalence of this LGMD.

No grant support is associated with preparation of this manuscript.

\section{REFERENCES}

1. Jarry J, Rioux MF, Bolduc V, Robitaille Y, Khoury V, Thiffault I, et al. A novel autosomal recessive limb-girdle muscular dystrophy with quadriceps atrophy maps to 11p13-p12. Brain 2007;130:368-380.

2. Mahjneh I, Jaiswal J, Lamminen A, Somer M, Marlow G, Kiuru-Enari $\mathrm{S}$, et al. A new distal myopathy with mutation in anoctamin 5. Neuromuscul Disord 2010;20:791-795.

3. Hicks D, Sarkozy A, Muelas N, Koehler K, Huebner A, Hudson G, et al. A founder mutation in Anoctamin 5 is a major cause of limbgirdle muscular dystrophy. Brain 2011;134:171-182.

4. Deschauer M, Joshi PR, Glaser D, Hanisch F, Stoltenburg G, Zierz S Muscular dystrophy due to mutations in anoctamin 5: Clinical and molecular genetic findings. Nervenarzt 2011;82:1596-1603.

5. Bolduc V, Marlow G, Boycott KM, Saleki K, Inoue H, Kroon J, et al. Recessive mutations in the putative calcium-activated chloride channel Anoctamin 5 cause proximal LGMD2L and distal MMD3 muscular dystrophies. Am J Hum Genet 2010;86:213-221.

6. Milenkovic VM, Brockmann M, Stohr H, Weber BH, Strauss O. Evolution and functional divergence of the anoctamin family of membrane proteins. BMC Evol Biol 2010;10:319.

7. Duran C, Hartzell HC. Physiological roles and diseases of Tmem16/ Anoctamin proteins: are they all chloride channels? Acta Pharmacol Sin 2011;32:685-692.

8. Mizuta K, Tsutsumi S, Inoue H, Sakamoto Y, Miyatake K, Miyawaki $\mathrm{K}$, et al. Molecular characterization of GDD1/TMEM16E, the gene product responsible for autosomal dominant gnathodiaphyseal dysplasia. Biochem Biophys Res Commun 2007;357:126-132.

9. Milone M, Liewluck T, Winder TL, Pianosi PT. Amyloidosis and exercise intolerance in ANO5 muscular dystrophy. Neuromuscul Disord 2012;22:13-15.

10. Adzhubei IA, Schmidt S, Peshkin L, Ramensky VE, Gerasimova A, Bork $\mathrm{P}$, et al. A method and server for predicting damaging missense mutations. Nat Methods 2010;7:248-249. 